Original Research Paper

\title{
On Containment and Radical Graphs of a Commutative Ring
}

\author{
Saba Al-Kaseasbeh \\ Department of Mathematics, Tafila Technical University, 66110, Tafila, Jordan
}

\section{Article history}

Received: 04-09-2016

Accepted: 29-12-2016

Email: kaseabeh@ttu.edu.jo
Revised: 16-11-2016

\begin{abstract}
In this study, we consider various graphs with the vertices being sets of ideals of a commutative ring with identity, $R$. How we define the edge set will vary according to distinct ideal theoretic properties. We apply a graphical approach to the study of ideal relationships in commutative rings and from this we obtain some properties of their graphs.
\end{abstract}

Keywords: Commutative Ring, Radical Ideal, Connected Graph, Complete Graph

\section{Introduction}

The study of commutative ring theory using the concepts of graph theory has attracted considerable attention in the last years. Different graphs have been high- lighting the interplay between graph theory and algebraic structures. An example of such interplay can be found in the annihilating ideal graph. The authors in (Behboodi and Rakeei, 2011), defined the annihilating ideal graph to be a graph whose vertex set is the set of ideals with nonzero annihilators and there is an edge between $I$ and $J$ if and only if $I J=0$. In (Chakrabarty et al., 2009), the authors introduced the intersection graph of ring in which vertices are non trivial ideals and there is an edge between $I$ and $J$ if and only if $I \cap J \neq 0$. In (Beck, 1988), Beck introduced the zero divisor graph where the set of vertices is the elements of $R$ and two elements $\mathrm{a}$ and $\mathrm{b}$ in the ring $\mathrm{R}$ are adjacent if $a b=0$. In (Anderson and Livingston, 1999), they introduced a refined definition of the zero divisor graph to give a good picture of the zero divisor structure of the ring. In the new definition the set of vertices is the set of nonzero zero divisors of the ring and two elements $a$ and $b$ in the ring $\mathrm{R}$ are adjacent if $a b=0$. In (Ye and $\mathrm{Wu}, 2012$ ) the author defined the comaximal ideal graph of a commutative ring $R$, whose vertice are the proper ideals of $R$ which are not contained in the Jacobson radical of $R$ and two vertices $I$ and $\mathrm{J}$ are adjacent if and only if $I+J=R$.

In this study, we explore two types of graphs that appear when we use different assumptions to define our edge set where the vertex set of the graph is the set of the ideals of the commutative ring with identity $R$. This paper continues with the main target of research in the field of ideal graphs. Our particular focus is on $\Omega_{1}$, where edge structure is determined by containment and $\Omega_{2}$, the radical ideal graph which are obtained from the commutative ring with unity.
First, we would like to recall some basic definitions and results from graph theory (Chartrand, 2006) and ring theory (Dummit and Foote, 2004), which have been used through out the paper.

A graph $G=(V, E)$ consist of a finite nonempty set of vertices denoted by $V(G)$ and a collection of unordered pair of distinct vertices of $\mathrm{G}$ called edges denoted by $E(G)$. The cardinality of the vertex set of a graph $\mathrm{G}$ is called the order of $\mathrm{G}$ denoted by $|V|$. The degree of any vertex $v$ of $G$ is the number of edges incident with vertex $v$. In a graph $G$, a finite alternating sequence of vertices and edges starting and ending with vertices such that each edge in the sequence is incident with the vertices following and preceding it is called a Walk. A path is an open walk in which no vertex appears more than once. A graph $G$ is connected if $G$ contains a $v_{1}-v_{2}$ path for every pair $v_{1}, v_{2}$ of distinct vertices of $G$. A graph $G$ is complete if every two distinct vertices of $G$ are adjacent.

We recall that an ideal $P$ is prime ideal if $P \neq R$ and if $r, s \in P$ implies $r \in P$ or $s \in P$. An ideal $M$ is a maximal ideal if $M \neq R$ and for any ideal $I \subseteq R$ with $M \subseteq I \subseteq R$ implies $M=I$ or $M=R$. We recall that an ideal is a radical if it is an intersection of primes, or equivalently, if $I=\sqrt{I}\left\{r \in R \mid r^{n} \in I\right.$ where $n$ is a positive integer $\}$.

\section{Main Results}

In this section, we introduce two types of graphs that appear when we use different assumptions to define our edge set, where the vertex set of the graph is the set of the ideals of the commutative ring with identity $\mathrm{R}$ and study its properties.

\section{Definition 2.1}

Let $R$ be a commutative ring with unity. We define the graph $\Omega_{1}$ if the vertex set is the set of the ideals of $R$ 
and two vertices $I$ and $J$ have an edge between them if and only if $I \subset J$.

By definition of our new graph, there is an edge between the zero ideal and any vertex in $\Omega_{1}$. Moreover, there is no loop in any vertex in $\Omega_{1}$.

\section{Theorem 2.1}

$\Omega_{1}$ is connected graph with diameter less than or equal 2 .

\section{Proof}

Of course, we have the path $I-(0)-J$.

\section{Theorem 2.2}

$\Omega_{1}$ is complete if and only if $R$ is an valuation ring.

\section{Proof}

Assume that $\Omega_{1}$ is complete graph. Then any two vertices of the graph $\Omega_{1}$ are connected. Let $I$ and $J$ be two ideals in $R$ (verticies in the graph). Since (as verticies) $I$ and $J$ have an edge between then, then $I$ and $J$ (as ideals) are comparable. Hence $\mathrm{R}$ is a valuation ring.

Now we suppose that $R$ is an valuation ring. Hence any two ideals are comparable and hence every two vertices are connected. hence, $\Omega_{1}$ is complete.

\section{Example 2.1}

Consider the ring $Z_{30}$. The set of ideals in $Z_{30}$ are $(0)$, (2), (3), (5), (6),(10) and (15). Figure 1 shows the graph $\Omega_{1}$ of the ring $Z_{30}$.

Of course, it is more interesting to ask questions concerning connectivity if (in the spirit of (Anderson and Livingston, 1999)) we modify our set of verticies for $\Omega_{1}$. In this sence, we define $\Omega_{1 *}$ to be the subgraph of $\Omega_{1}$ with verticies consisting of all nonzero ideals of $R$. We begin with a lemma.

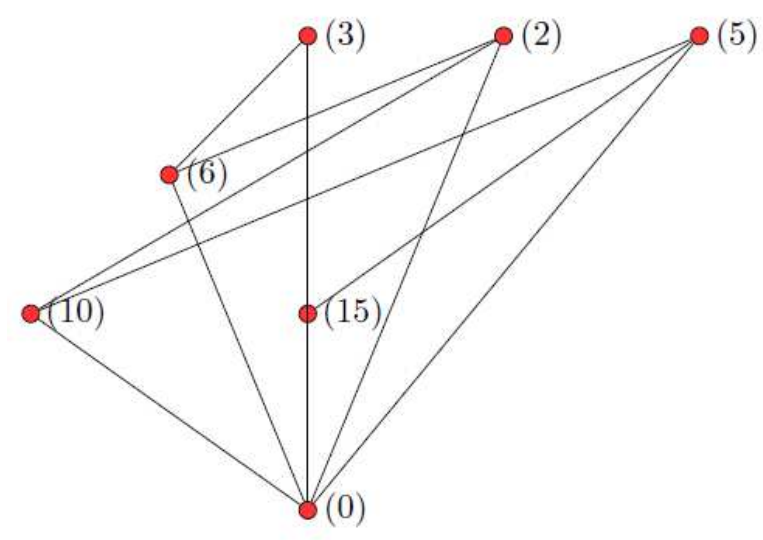

Fig. $1 . \Omega_{1}\left(Z_{30}\right)$

\section{Lemma 2.1}

Let $R$ be commutative with identity and $M_{1}, M_{2}, M_{3}$ be distinct maximal ideals of $R$. If $M_{1} M_{2}=0=M_{2} M_{3}$ then $M_{2}=0$.

Put loosely, this means that no maximal ideal can annihilate two distinct maximal ideals.

\section{Proof}

Since $M_{1}$ and $M_{3}$ are distinct maximal ideals, then we can find $m_{1} \in M_{1}$ and $m_{3} \in M_{3}$ such that $m_{1}+m_{3}=1$. Multiplying this by a nonzero $m_{2} \in M_{2}$, we obtain that $m_{2} m_{1}+m_{2} m_{3}=m_{2}=0$ which is our desired contradiction.

What follows is a couple of theorems to explain the structure (connectivity) of the graph $\Omega_{1 *}$. It is interesting that the case of two maximal ideals needs to be handled as a special case.

\section{Theorem 2.3}

Let $R$ be a commutative ring with 1 and $\operatorname{Max} \operatorname{Spec}(R)$ $=M$ the collection of maximal ideals of $R$. The graph $\Omega_{1 *}$ is connected if $|M| \neq 2$. Additionally $\operatorname{diam}\left(\Omega_{1^{*}}\right) \leq 2$ if $|M|$ $=1$ and $\operatorname{diam}\left(\Omega_{1 *}\right) \leq 6$ if $|M|>2$.

\section{Proof}

Note that if $|M|=1$ then there is a unique maximal ideal $M$ and so given ideals $0 \neq I, J \subset R$, we have the path $I$ $M-J$. This also shows that the diameter does not exceed 2.

We now assume that there at least three maximal ideals. Assume that we have $0 \neq I, J \subset R$. We will assume that $I \subseteq M_{1}$ and that $\mathrm{J} \subseteq M_{2}$. If $M_{1} \cap M_{2} \neq 0$, we select a nonzero element, a in this intersection and note that we have the path of length 4 :

$$
I=M_{1}-(a)=M_{2}-J
$$

Now, we assume that $M_{1} \cap M_{2}=0$. By the previous lemma, we now have that $M_{3}$ must have nontrivial intersection with both $M_{1}$ and $M_{2}$. We therefore select $m_{1} \in \mathrm{M}_{1} \cap M_{3}$ and $m_{2} \in M_{2} \cap M_{3}$ and this gives the following path of length 6 :

$$
I=M_{1}-\left(m_{1}\right)=M_{3}-\left(m_{2}\right)-M_{2}-J
$$

We now examine the outstanding case where $|M|=2$.

\section{Theorem 2.4}

Let $\mathrm{R}$ be a commutative ring with 1 that has precisely two maximal ideals $M_{1}$ and $M_{2}$, then $\Omega_{1 *}$ is connected if and only if $M_{1} M_{2} \neq 0$ and in this case $\operatorname{diam}\left(\Omega_{1 *}\right) \leq 4$.

\section{Proof}

If $I \subseteq M_{1}, J \subseteq M_{2}$ and $0 \neq a \in M_{1} M_{2}$, we have the path of length 4 : 


$$
I-M_{1}-(a)-M_{2}-J
$$

We now present a new graph that is specified by ideal theoretic properties and give some results for our new graph.

\section{Definition 2.2}

Let $R$ be a commutative ring with unity. We define the graph $\Omega_{2}$ if the vertex set is the set of ideals of $R$ and two vertices $I$ and $J$ have an edge between them if and only if $\sqrt{I}=\sqrt{J}$.

\section{Theorem 2.5}

The connected components of the graph $\Omega_{2}$ are in one to one correspondence with the distinct intersections of prime ideals in $R$.

\section{Proof}

It is well-known that an ideal is radical if and only it is an intersection of prime ideals.

\section{Corollary 2.1}

Let $R$ be a commutative ring of Krull dimension $n$. The number of connected components of $\Omega_{2}$ is bounded below by $n+1$ and if $\operatorname{dim}(R)=n<\infty$ then equality holds if and only if the prime ideals are linearly ordered.

\section{Proof}

If $P_{0} \nsubseteq P_{1} \nsubseteq \ldots \nsubseteq P_{n}$ is a chain of prime ideals then each $P_{i}$ corresponds to a different connected component, which establishes the first statement.

If $\operatorname{dim}(R)=n$ then there exists a chain of prime ideals of $R$ :

$$
P_{0} \nsubseteq P_{1} \nsubseteq \ldots \nsubseteq P_{n}
$$

If the primes are not linearly ordered then there is a prime ideal $Q$ that does not compare to some $P_{i}$ above. If this case the ideal $P_{i} \cap Q$ is a radical ideal that defines another connected component.

\section{Corollary 2.2}

Let $R$ be a valuation domain of Krull dimension $\mathrm{n}$. Then $\Omega_{2}$ has precisely $n+1$ connected components.

\section{Proof}

This follows from the proof of corollary 2.1.

\section{Example 2.2}

Consider the ring $Z_{100}$. The set of ideals in $Z_{100}$ are (0), (2), (4), (5), (10), (20), (25) and (50). By definition of the radical of an ideal $I$ in $R$, its easy to see that, $\sqrt{(2)}=(2), \sqrt{(3)}=(3), \sqrt{(4)}=(2), \sqrt{(5)}=(5)$, $\sqrt{(10)}=(10), \sqrt{(20)}=(10), \sqrt{(25)}=(5), \sqrt{(50)}=(10)$. It is clear that the zero ideal will not adjacent with any vertex in $\Omega_{2}$. Figure 2 shows the graph $\Omega_{2}$ of the ring $Z_{100}$.

\section{Theorem 2.6}

Let $R$ be a commutative ring with 1 . The following conditions are equivalent:

\section{1. $\Omega_{2}$ is connected.}

2. $\Omega_{2}$ is complete.

3. $R$ is quasilocal and $\operatorname{dim}(R)=0$

Proof. (2) implies (1) is immediate as any complete graph is connected. For (1) implies (3), we note that if $\Omega_{2}$ is connected then $\sqrt{I}=\sqrt{J}$ for all ideals in $R$. In particular $\sqrt{(0)}$ is the radical of every ideal of $R$. Hence $R$ has a unique prime ideal and hence is quasilocal and of dimension 0. Finally, for (3) implies (2), we note that if $R$ has a unique prime, then $\sqrt{I}=\sqrt{J}$ for all proper ideals $I, J \subset R$ and hence $\Omega_{2}$ is complete.

We end with a last observation that we find interesting. By constructing appropriate valuation domains, we can create graphs $\Omega_{2}$ with any arbitrary number of connected components, but such valuation domains (of dimension greater than 1) are necessarily non-Noetherian. The Noetherian case is somewhat more restrictive.

\section{Theorem 2.7}

Let $\mathrm{R}$ be Noetherian and suppose $\Omega_{2}(R)$ has only finitely many connected components. Then $\operatorname{dim}(R) \leq 1$ and $\mathrm{R}$ has only finitely many prime ideals. What is more:

1. If $R$ has $n$ prime ideals, then $\Omega_{2}(R)$ has no more than $2^{n}-1$ connected components.

2. If $R$ is an integral domain with n prime ideals then $R$ has precisely $2^{n}-1$ connected components.

\section{Proof}

It is well- known that if $R$ is Noetherian of Krull dimension greater than 1 , then $\mathrm{R}$ has in finitely many primes; this establishes the first statement.

To justify (1), we note that the distinct radical ideals consist of the distinct intersections of the $\mathrm{n}$ prime ideals. Counting the possible intersections by the number of ideals being intersected, we obtain:

$$
\left(\begin{array}{l}
n \\
1
\end{array}\right)+\left(\begin{array}{l}
n \\
2
\end{array}\right)+\cdots+\left(\begin{array}{l}
n \\
n
\end{array}\right)=2^{n}-1
$$




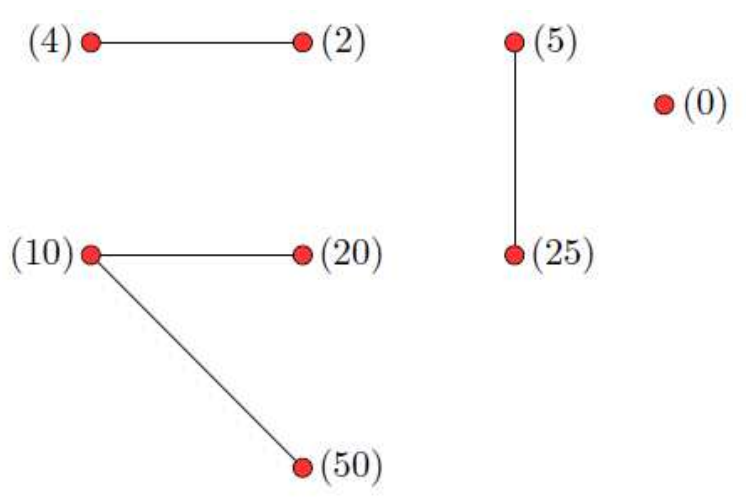

Fig. $2 . \Omega_{2}\left(Z_{100}\right)$

This number of functions as the claimed are upper bound in (1).

If $R$ is a domain, then (0) is a prime (and intersections involving (0) are, of course, trivial). If $\mathrm{R}$ is 0 dimensional then $R$ is a field and the only prime (radical) ideal is $(0)$ and hence the formula is verified. In the final case that $\operatorname{dim}(R)=1$, then there are precisely $n-1$ nonzero primes and every distinct subset gives a unique radical ideal. Applying the formula from (1) and adding the ideal $(0)$, we obtain precisely $\left(2^{n-1}-1\right)+1=2^{n-1}$ connected components.

\section{Conclusion}

In this study, we explored two types of graphs that appear when we use different assumptions to define our edge set, where the vertex set of the graph is the set of the ideals of the commutative ring with identity $R$. We concentrated on $\Omega_{1}$, where edge structure is determined by containment and $\Omega_{2}$, the radical ideal graph which are obtained from the commutative ring with unity. We gave some results and justifications for our investigated graphs. Also, we provided the reader with some examples that give a clear view of $\Omega_{1}$ and $\Omega_{2}$.

\section{Acknowledgement}

I would like to thank Prof. Jim Coykendall for suggesting the research problem and anonymous referee for their very constructive suggestions that contributed to the improvement of this version of the paper.

\section{Ethics}

This article is original and contains unpublished material. The corresponding author addresses any ethical issues that may arise after the publication of this manuscript.

\section{References}

Anderson, D.F. and P.S. Livingston, 1999. The zerodivisor graph of a commutative ring. J. Algebra, 217: 434-447. DOI: 10.1006/jabr.1998.7840

Beck, I., 1988. Coloring of commutative rings. J. Algebra, 116: 208-226. DOI: 10.1016/0021-8693(88)90202-5

Behboodi, M. and Z. Rakeei, 2011. The annihilatingideal graph of commutative rings I. J. Algebra Applic., 10: 727-739. DOI: $10.1142 / \mathrm{S} 0219498811004896$

Chakrabarty, I., S. Ghosh, T.K. Mukherjee and M.K. Sen, 2009. Intersection graphs of ideals of rings. Discrete Mathemat., 309: 5381-5392. DOI: $10.1016 /$ j.disc.2008.11.034

Chartrand, G., 2006. Introduction to Graph Theory. 1st Edn., Tata McGraw-Hill Education, New Delhi, ISBN-10: 0070616086, pp; 449.

Dummit, D.S. and R.M. Foote, 2004. Abstract Algebra. 3rd Edn., Wiley, Hoboken, ISBN-10: 0471452343, pp: 932.

Ye, M. and T. Wu, 2012. Co-maximal ideal graphs of commutative rings. J. Algebra Applic., 11: 1250114-1250114. DOI: $10.1142 / \mathrm{S} 0219498812501149$ 